\title{
Actitud ante las Fake News: Estudio del caso de los estudiantes de la Universidad del País Vasco
}

\author{
Facing Fake News: The case of the students of the University of \\ the Basque Country
}

\author{
Mendiguren, T., Pérez Dasilva, J. y Meso Ayerdi, K. 1 \\ Recibido: 24-09-2019 - Aceptado: 25-01-2020 \\ DOI: https://doi.org/10.26441/RC19.1-2020-A10
}

\begin{abstract}
RESUMEN: Internet y las redes sociales se han convertido en la vía de información más atractiva y accesible para los jóvenes. Objetivos. Examinar las conductas de los jóvenes universitarios ante las fake news y pulsar su opinión sobre este fenómeno. Metodología. Encuestas semiabiertas realizadas principalmente al alumnado Periodismo de la Universidad del País Vasco. En una segunda fase se amplía el estudio a estudiantes de otras áreas de conocimiento. Resultados. Los universitarios se informan principalmente en el entorno online y un alto porcentaje confiesa haber caído en la trampa de las fake news en alguna ocasión. Conclusiones y discusión. Se detectan un alto índice de alerta por parte de los universitarios ante las noticias falsas y algunas diferencias, según el área de conocimiento, de las temáticas que consideran más invadidas por las fake news.
\end{abstract}

Palabras clave: noticias falsas; estudiantes universitarios; millenials; Generación Z.

\begin{abstract}
The Internet and social networks have become the most attractive and accessible information channel for young people. Objectives. Examine the behavior of university students when facing fake news and their opinion on this phenomenon. Methodology. Semi-open surveys conducted mainly for Journalism students from the University of the Basque Country. In a second phase, the study was extended to students from other areas of knowledge. Results. Online media use is commonplace for university students and a high percentage of them confess to have been fooled by fake news on at least one occasion. Conclusions and discussion. The students consider they have a high rate of alert when facing fake news. There are some differences, according to the area of knowledge, of the subjects that they consider more invaded by the fake news.
\end{abstract}

Keywords: fake news; students; university students; millennials; Z Generation.

\footnotetext{
${ }^{1}$ Terese Mendiguren es Doctora en Ciencias de la Información por la Universidad del País Vasco. Actualmente imparte la asignatura de Locución Informativa en el Grado de Periodismo, así como en dos másteres profesionales de Periodismo Multimedia de la UPV-EHU. Es secretaria académica del Departamento de Periodismo II. Terese. mendiguren@ehu.eus, http://orcid.org/0000-0003-3092-6608

Jesús Pérez Dasilva es Doctor en Ciencias de la Información por la Universidad del País Vasco. Es profesor de las asignaturas Ziberkazetaritzako Idazkera y Redacción Ciberperiodística en la Facultad de Ciencias Sociales y de la Comunicación de la Universidad del País Vasco. jesusangel.perez@ehu.eus, https://orcid.org/0000-00023383-4859

Koldobika Meso Ayerdi es Doctor en Periodismo por la Universidad del País Vasco. Asimismo, Máster en Periodismo de El Correo/UPV-EHU. Es profesor de la asignatura Redacción Ciberperiodística e imparte Bases teóricas y metodología de la investigación en ciberperiodismo en el Máster en Comunicación Social en la Facultad de Ciencias Sociales y de la Comunicación de la Universidad del País Vasco. koldo.meso@ehu.eus, http://orcid. org/0000-0002-0400-133X
} 


\section{Introducción}

La proliferación de noticias falsas es un problema que afecta a la ciudadanía en general y los jóvenes en particular, dada la tendencia de los mismos a emplear las redes y aplicaciones sociales para mantenerse informados (Lenhart et al., 2010). Según un estudio sobre la capacidad de los jóvenes para evaluar mensajes y parcialidad en la información en Internet realizado por el grupo de investigación Stanford History Education Group realizado en 2016, la mayoría de los estudiantes no saben cuándo las noticias son falsas. La capacidad de los jóvenes para discernir entre las informaciones provenientes de la esfera online que carecen de credibilidad y las que sí la tienen, es escasa:

"Muchos asumen que, debido a que los jóvenes son fluidos en las redes sociales, son igualmente conocedores de lo que encuentran allí ... nuestro trabajo muestra lo contrario" (Wineburg y McGrew, 2017).

Las personas que han crecido con Internet y las nuevas tecnologías digitales se conocen como nativos digitales, pero las conclusiones del citado estudio advierten de que estos nativos a menudo están aún más perdidos en el mundo de la información online que los adultos no nativos. En este contexto, algunos medios sociales están tomando medidas para combatir las noticias falsas después de recibir duras críticas por permitir que las historias con información fraudulenta circulen en sus plataformas. El director ejecutivo de Facebook, Mark Zuckerberg, consideraba que su empresa estaba destinada a servir como una compañía de tecnología en lugar de un medio de comunicación, pero el creciente papel de esta red social en la distribución de noticias ha hecho que esa idea cambie ${ }^{2}$.

Esta investigación ${ }^{3}$ pulsa la opinión y muestra la conducta de los estudiantes universitarios de la Universidad del País Vasco, ante las noticias falsas o lo que conocemos como fake news. Se trata de ver en qué proporción y de qué manera se enfrentan estos jóvenes a todas las voces presentes en las webs de noticias y redes sociales.

\section{Las fake news se cuelan en los medios}

Aunque el término fake news ha sido popularizado recientemente, la propagación de medias verdades y su influencia en la política ha sido muy estudiada (Berinsky, 2017; Brandzaeg et al., 2017; Fisher, 2016), así como la forma en la que se propagan los rumores (Friggeru et. al, 2014). Por tanto, la proliferación de noticias falsas no es un fenómeno nuevo, pero sí lo es la magnitud que hoy en día pueden llegar a adquirir en el entorno online. La expresión fake news no puede ser traducida literalmente porque si son noticias falsas, no son noticias, ya que "en el periodismo, en principio, no existe noticia falsa, tanto que una de las reglas es la verificación de los hechos antes de la publicación" (Parreira, 2019). La expresión abarca varias categorías y todas amenazan a la calidad del periodismo:

"Noticias fraudulentas o frágiles; información falsa (en general con fuentes forjadas), manipulada, adulterada o fabricada o "plantada" (con la intención de engañar); (que no se puede ver ni mal, sino que, a veces, fuera de contexto, para causar daños [mal informados]; noticias antiguas viejas; sensacionalismo (propio de los tabloides) mentiras, maquillajes, rumores, hechos alternativos etc. (Parreira, 2019)

Las denominadas fake news pueden definirse además como artículos de noticias que son intencional y verificablemente falsos, y que podrían inducir a error a los lectores (Allcot y Gentzkow, 2017).

\footnotetext{
2 https://www.csmonitor.com/Technology/2016/1122/Many-teens-can-t-tell-real-news-from-fake-study-finds

${ }^{3}$ Este trabajo forma parte del proyecto "Audiencias activas y viralización y transformación de los mensajes periodísticos" (CSO2015-64955-C4-4-R), financiado por el Plan Nacional del I+D+i, del Ministerio de Economía y Competitividad, y por el Fondo Europeo de Desarrollo Regional (Feder).
} 
"En cada época se han utilizado los medios existentes para difundir propaganda y falsedades, para informar o desinformar. Actualmente, la web ha cambiado la dinámica de la transmisión de la información y los hechos se mezclan con verdades y mentiras para generar mezclas informativas que impulsan y multiplican la especulación y el entretenimiento.” (Parra y Oliveira, 2018)

La difusión de las fake news se beneficia de las prácticas de compartición entre usuarios humanos y bots computarizados que pueden llegar a amplificar exponencialmente el poder de las campañas de desinformación (Allcot y Gentzkow, 2017). En este contexto, los usuarios de Internet son vulnerables a quedar atrapados en un círculo vicioso de noticias falsas y desinformación debido a que Google y Facebook usan filtros algorítmicos para dictar la información que los usuarios ven primero en sus búsquedas y noticias (Pariser, 2011). El último informe sobre consumo de noticias de los usuarios españoles publicado por la Universidad de Navarra ${ }^{4}$ recoge que las redes sociales y los blogs crecen como fuente preferible de noticias, superando a las webs y aplicaciones de diarios y revistas. Este estudio dirigido por Alfonso Vara demuestra que cuanto más joven es la audiencia, más preferencia muestra por las redes sociales.

Por otro lado, la rapidez siempre ha sido un componente valorado del periodismo. La audiencia quiere estar informada sobre los cambios relevantes en su mundo lo antes posible. Los periodistas deben tener en cuenta que "obtener la primicia es un desafío, pero ser riguroso o preciso es una necesidad para sobrevivir en la práctica del periodismo de calidad" (Palomo, 2018).

La difusión de noticias y artículos falsos se ha convertido en una práctica habitual en la era de las redes sociales. Estas plataformas se configuran como el ecosistema perfecto para la expansión de contenidos engañosos cargados de intencionalidad, ya sea política, ideológica o económica.

\section{Millenials, Generación Zeta y la esfera mediática}

Generalmente se considera que el término millenial se aplica a las personas que alcanzaron la edad adulta alrededor del cambio de siglo XXI. También conocidos como Generación Y, constituyen la franja demográfica que sigue directamente a la Generación X. Representan la última generación del siglo XX y la primera generación verdaderamente digital desde su existencia. Aproximadamente el $50 \%$ de la población mundial tiene menos de 30 años (Foro Económico Mundial, 2017), la llamada Generación del Milenio o los millenials ocupará en el año 2030 la mayor parte de los cargos de responsabilidad en todos los ámbitos laborales existentes (Alberto Notario, 2018).

Esta generación también está marcada por la transición de un milenio a otro, de ahí el nombre. Los millenials son los sucesores de dos generaciones influyentes, los baby boomers, personas nacidas entre el año 1946 y 1964, así como la "generación X", personas nacidas entre el año 1965 y 1979 (Plazibat et al, 2017). Algunas fuentes opinan que pertenecen a esta generación todos los nacidos entre los años 1975 y 1995 (Alberto Notario, 2018); de acuerdo con otras, los millennials han nacido en el periodo entre 1985 y 2010 (Wells, 2015). Al margen de la diversidad de criterios con los que se puede delimitar demográficamente el término, resulta interesante destacar que sí existe un cierto consenso sobre las características de la Generación del Milenio como un grupo relativamente homogéneo (Alberto Notario, 2018). A diferencia de las generaciones anteriores, los millenials son bastante expresivos al mostrar sus preferencias y actitudes, algo que logran fácilmente utilizando Internet, las redes sociales y los teléfonos móviles (Plazibat et al, 2017). En términos generales, los millennials están considerados como personas autosuficientes, trabajadores, optimistas sobre el futuro, tolerantes y abiertos al cambio (Leahy et al 2011), al mismo tiempo que se destacan sus

\footnotetext{
${ }^{4}$ http://www.noticiasdenavarra.com/2017/06/22/ocio-y-cultura/comunicacion/los-medios-periodisticos-siguen-siendo-los-mas-confiables-por-los-internautas-frente-a-las-redes-sociales
} 
grandes aptitudes para realizar de forma simultánea varias tareas (Anderson y Rainie, 2012). Precisamente debido a estas características y su estilo de vida conectado a las redes, las personan que forman parte de esta generación se influyen mutuamente, así como a ciudadanos de otras franjas de edad. Estos patrones de comportamiento resultan muy interesantes para los especialistas en marketing (Plazibat et al, 2017). También se les considera como una generación con facilidad para adquirir conocimientos de forma autodidacta (Hidalgo Pérez, 2017) y con una gran conciencia sobre cuestiones sociales y medioambientales (Foro Económico Mundial, 2017). En cualquier caso, la frontera entre las etiquetas otorgadas a los jóvenes y su papel en la sociedad suele ser difusa. Según Schroer (2008), los niños y adolescentes que han nacido entre los años 1995 y 2012 forman parte de la Generación Z. También se han utilizado otros nombres para referirse a este grupo social, como Generación V(vitual), Generación C (comunidad), Generación Silenciosa, Generación de Internet o Generación Google (Fernandez Cruz y Fernández Díaz, 2016). "Los primeros representantes de la Generación $Z$ están saliendo de la universidad y empiezan a incorporarse a un mercado de trabajo en transformación" (Magallón, 2016). Los rasgos comunes que los define son las Tecnologías de la Información y la Comunicación (TIC), la interacción, la rapidez, la impaciencia y la resiliencia, entre otros (Fernández Cruz y Fernández Díaz, 2016).

Respecto al consumo de información por parte de los jóvenes, la mayoría de las personas menores de 30 años no se suscribe a los periódicos ni consume habitualmente las noticias de televisión y radio (Marchi, 2012). Algunos investigadores interpretan estas tendencias como indicios de que los jóvenes de hoy no están interesados en las noticias y tienen una mentalidad menos cívica que las generaciones anteriores (Jones, 2008). Otros sostienen que el decreciente consumo de los medios de comunicación tradicionales no necesariamente significa que los jóvenes no estén interesados en las noticias o la política (Marchi, 2012). Parece que los usuarios más jóvenes valoran el hecho de que las noticias estén continuamente disponibles para mantenerse informados sobre las historias más relevantes o curiosas, y poder compartirlas. Algo similar ocurre con las tradicionales formas de consumo de la televisión.

"Los sectores más jóvenes de la población -millennials y generaciones venideras de menor edadmuestran un comportamiento audiovisual que prioriza los servicios siempre conectados y personalizables, frente a las ofertas rígidas y cerradas.” (Guerrero Pérez, 2018)

Tienen menos necesidad de consumir medios profesionales debido a la disponibilidad de alternativas gracias al amplio abanico de sitios web gratuitos y la tendencia al consumo a través del móvil. Este declive en el uso de los medios informativos tradicionales por parte de la población más joven ha sido frecuentemente objeto de investigación académica internacional (Banaji y Buckingham 2013, Pasek et al., 2006). Según algunos autores la mayor parte de las noticias se considera aburrida e irrelevante por parte de los jóvenes, que reclaman un consumo rápido de la información (Marchi 2012). Sin embargo, la imagen estándar de los jóvenes que solo están interesados en las noticias rápidas no es del todo cierta. Investigaciones previas a la era del periodismo móvil y las tablets muestran que los usuarios más jóvenes no solo quieren noticias rápidas, sino también profundidad y un tipo de periodismo más lento (Costera Meijer. 2007, 112). Por otro lado, los jóvenes actualmente se esfuerzan por encontrar noticias procedentes de fuentes fiables y basadas en hechos objetivos (Alberto Notario, 2018) y sienten la responsabilidad de frenar la difusión cada vez mayor de las llamadas fake news (Foro Económico Mundial, 2017). En este sentido, y según este Foro Económico Mundial (2017), los jóvenes son cuidadosos con la información que comparten en Internet y tratan de difundir información fiable. Tienen en cuenta los "sitios web certificados" (61.8\%), la reputación de la publicación $(53,6 \%)$ o las voces expertas $(48,4 \%)$. El citado estudio recoge que el $49.5 \%$ de los jóvenes respondieron "no" a la pregunta: “Alguna vez ha compartido un artículo de noticias o una noticia en Internet o en las redes sociales que luego descubrió que eran falso?" y concluye que se trata de una generación que se preocupa por la credibilidad de la información que consume. 


\section{Objetivos, hipótesis y metodología}

Teniendo en cuenta que una gran mayoría de los usuarios más jóvenes de la esfera mediática no tiene la necesidad de consumir medios profesionales debido a la disponibilidad de alternativas, sitios web gratuitos y la tendencia al consumo a través del móvil, esta investigación tiene por objetivo conocer la actitud de los estudiantes universitarios ante las Fake News. Concretamente, el grupo de investigación que ha llevado a cabo este estudio se planteaba las siguientes cuestiones: ¿Saben nuestros estudiantes detectar las Fake News? ¿Se consideran personas con criterio para distinguir una noticia fiable de otra que no lo es? ¿Consideran que pertenecen a una generación mejor preparada en ese sentido que la anterior? ¿Cómo verifican una información cuando sospechan que carece de rigor? ¿Cuál es la vía de difusión de noticias de la que menos se fían? ¿Cuánta credibilidad otorgan a los medios convencionales? Partiendo de los objetivos específicos de responder a las cuestiones planteadas, la investigación se ha realizado en dos fases.

Los datos obtenidos en la primera fase provienen de un análisis cuantitativo y cualitativo, mediante encuesta, sobre la actitud de los estudiantes de periodismo de la Universidad del País Vasco ante las Fake News.

A través de un cuestionario, se han registrado los datos relacionados con el perfil del encuestado (edad, género, curso, área de conocimiento) y trece preguntas en torno al grado de conocimiento del alumnado sobre el fenómeno de las Fake News, la percepción y actitud de los propios estudiantes ante las mismas, las áreas temáticas ${ }^{5}$ donde creen que destacan las noticias falsas, las vías a través de las cuales consideran que se difunden, la consideración ética de los medios convencionales en torno a la información que publican, para tratar de combatir la proliferación de noticias falsas. El cuestionario combina los estilos de pregunta cerrada, abierta y semiabierta. Para la realización del cuestionario se ha consultado la literatura de investigación en torno al método de la encuesta (Gil, 2001), sobre todo en el contexto de los jóvenes y las TIC (López, 2011; Del Barrio y Ruiz, 2017), pero el cuestionario ha debido ser diseñado ad hoc para este estudio.

En esta primera fase se han registrado más de 120 los alumnos y alumnas entrevistados de entre $20 \mathrm{y}$ 22 años (entre segundo y tercero de la carrera de Periodismo). Los estudiantes de periodismo fueron elegidos como objeto de estudio porque son un subconjunto social significativo de consumidores de medios de comunicación, dada la formación que están recibiendo, y constituyen en cierta medida parte del futuro vértice profesional del tejido mediático. Partimos de la hipótesis de que están familiarizados con los procesos de verificación de las fuentes y los datos, y se les presupone cierto grado de preparación para enfrentarse a la identificación de noticias falsas. Las encuestas tuvieron lugar entre abril y mayo del 2018.

Tras analizar las respuestas del cuestionario, la investigación derivó en una segunda fase: comparar estos resultados con los que se obtendrían en una segunda vuelta de encuestas a estudiante de otras áreas de conocimiento. Con ese fin se pasaron las mismas encuestas a 200 alumnos y alumnas del Grado de Ciencias Políticas, Económicas, Física, Medicina y Bellas Artes. Se escogieron estas áreas de conocimiento por tratarse, en líneas generales, de carreras ligadas a las secciones temáticas recurrentes en los medios de comunicación de información general (política, economía, ciencias, salud,

\footnotetext{
${ }^{5}$ Las áreas temáticas que los encuestados deben elegir en función de dónde creen que abundan más las Fake News se han dividido en política, economía, temas sociales, salud/medio ambiente, cultura y otros. Los temas sociales en este caso abarcarían aquellos relacionados con vivienda, inmigración, cuestiones de género o inclusión social, disgregando salud/medio ambiente y cultura, con el fin de poder establecer, en una segunda fase de la investigación, relaciones específicas entre éstas últimas variables temáticas y las áreas de conocimiento de los estudiantes de Medicina/Física y Bellas Artes.
} 
cultura...). Para el análisis de los resultados y su cálculo estadístico se ha empleado el lenguaje de programación no procedimental que ofrece Microsoft Excell.

Las encuestas tuvieron lugar entre septiembre y noviembre del año 2018. En este caso, la hipótesis de partida era que estos estudiantes no están tan bien preparados como los de periodismo para detectar las noticias falsas y que los alumnos de cada una de las áreas consideran que es en su área de conocimiento donde se registran más casos de Fake News. En este sentido, los alumnos de económicas atribuirían a las informaciones sobre economía una mayor susceptibilidad para ser invadidas por informaciones falsas, a su vez los de medicina la atribuirían a los temas de salud, los de física a las ciencias y los de Bellas Artes a las informaciones de cultura.

En ambas fases se ha recurrido a un tipo de muestra no probabilística, que se suele emplear en los análisis con gran peso cualitativo y cuando la finalidad del estudio no es generalizar los resultados a toda la población, sino estimar relaciones entre variables y recopilar datos comparativos y descriptivos. Se trata de determinar la muestra bajo el criterio del investigador tomando como referencia los objetivos de la investigación. Lo que Babbie (1999:174) denomina muestreo deliberado o crítico. La muestra no probabilística intencional suele ser pequeña y heterogénea. El investigador la selecciona procurando que esta sea "representativa y, por consiguiente, su representación depende de su intención" (Visauta, 1989:155).

\section{Resultados}

\subsection{Investigación principal (primera fase)}

Tras registrar los datos obtenidos en las encuestas se ha constatado que la prensa digital es la vía más empleada por los estudiantes de periodismo para mantenerse informados. Sorprendentemente, a pesar de ser una expresión muy en auge en tanto en el ámbito periodístico como en el ámbito de la investigación en comunicación social, no todos los alumnos y alumnas consultados conocían la expresión o el concepto conocido como fake news. El 17\% de las personas consultadas no conocían el término, pero la gran mayoría sí había oído hablar de él.

Algunos las definen como "noticias falsas que tienen la intención de influir en la opinión de las personas", "noticias falsas, normalmente difundidas por redes sociales, con el fin de manipular la opinión popular para los intereses de quien las extiende" o "Noticias falsas y rumores creadas con el propósito de cambiar una actitud, de persuadir", haciendo hincapié en el objetivo de cambiar la opinión popular. En otros casos subrayan el concepto de falsedad cuando aluden a definiciones como "noticias con información falsa", "noticias con información falsa que se hacen tan conocidas que muchos las acaban dando por verdaderas sin contrastarlas siquiera", "noticias falsas que se propagan como reales", "noticias que son bulos", "noticias que no dicen totalmente la verdad" o "son noticias que se dan por verdaderas sin serlo, porque se apoyan en algún dato que es veraz". Estas dos últimas definiciones resultan interesantes porque subrayan uno de los ingredientes fundamentales del éxito para la propagación de las fake news: el hecho de que se apoyen en algunos datos o evidencias reales.

El $82 \%$ de los alumnos encuestados reconoce que en alguna ocasión ha dado por verídica una información que no lo era. Dentro de este porcentaje destaca Facebook como la vía a través de la cual recibieron ese contenido falso. Al tratarse de una pregunta semiabierta, algunos encuestados han especificado las plataformas Twitter y Facebook, pero otros han atribuido a las redes sociales en general esa vía de propagación del contenido recibido (gráfico 1). Por tanto, Facebook podría estar incluso más arriba en ese pódium de distribuidores de noticias falsas en el caso que nos ocupa. En cualquiera caso, Facebook es la red social más citada a la hora de detectar de dónde provenía la 
noticia falsa, en el caso de los estudiantes que afirmaban haber creído en alguna ocasión una información que no era cierta.

Gráfico 1. Procedencia de la noticia falsa, considerada "verdadera"

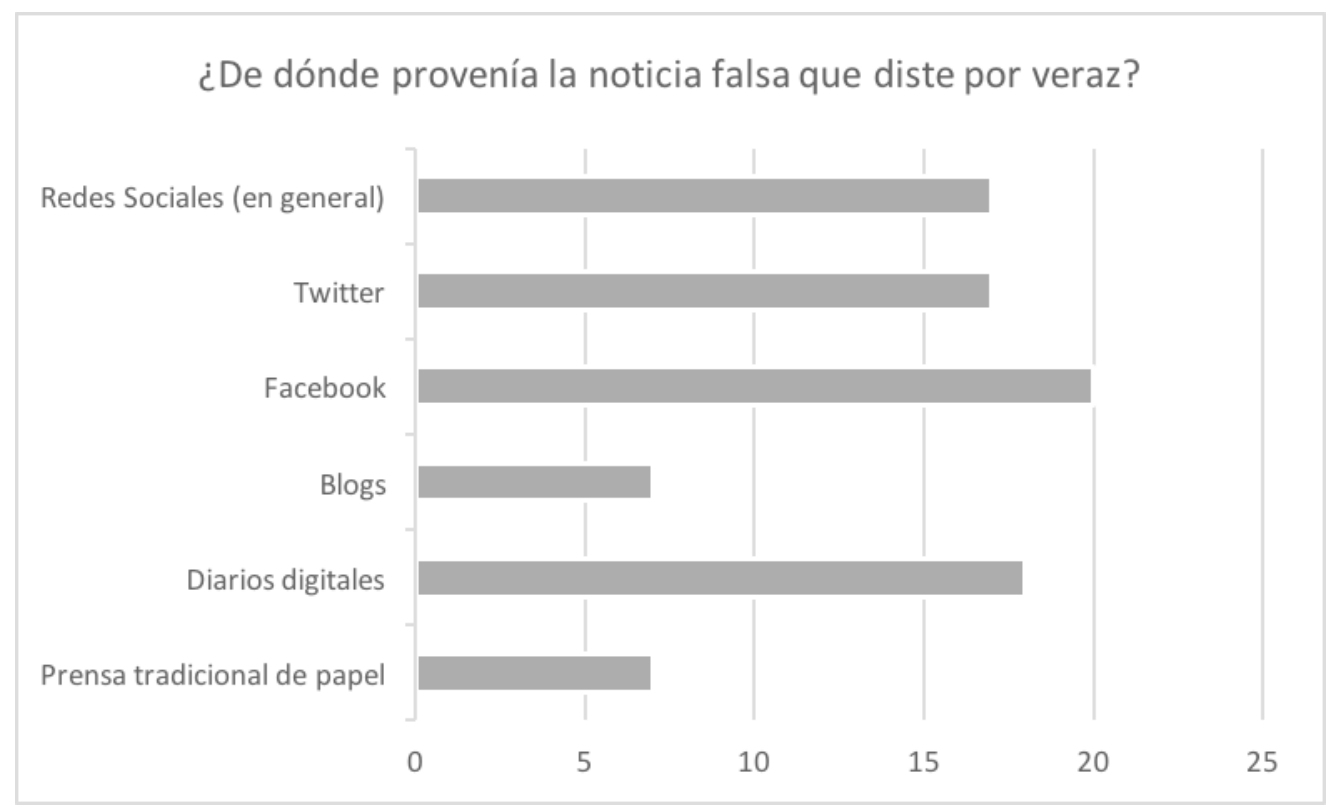

Fuente: elaboración propia

Ante la tesitura de encontrarse frente a una información dudosa las estrategias para salir de dudas son diversas. En algunos casos tratan de contrastar la información buscando "en medios tradicionales", "en diarios digitales que tienen más reputación y resultan más fiables", tratan de mirar "otras fuentes más creíbles", "en diarios profesionales" o en medios que consideras "fiables". Google también se convierte en aliado para contrastar las informaciones dudosas, ya que algunos afirman que acuden al citado buscador "con las palabras clave que son objetivas, para contrastar" o en páginas como "maldito bulo". Además, se fijan en el tipo de web que publica la noticia y la fecha de publicación. Por último, únicamente dos alumnos han citado Twitter como vía para contrastar las noticias que consideran dudosas.

El $80 \%$ de las personas consultadas creen que tiene criterio para distinguir una información o noticia fiable de otra que no lo es. Por otro lado, el 74\% de los encuestados admite que hoy en día desconfía más, respecto a unos años atrás, de la información que recibe a diario desde diversos canales. Esto se debe principalmente a que "cada vez más, con el auge de los medios digitales y sobre todo, redes sociales, la gente tiene más oportunidad para difundir los contenidos y mensajes que quieren, sean verdaderos o falsos". Consideran que ahora "hay más información falsa" porque "cualquiera puede publicar una información" y existe "facilidad de hacer y propagar fake news en las redes sociales".

Un alto porcentaje de encuestados dice ser precavido con la información que recibe. Muchos de ellos relacionan esta apreciación con el hecho de ser estudiantes de periodismo. Esta interpretación se extrae de las respuestas que afirman "he aprendido a comparar", "desde que estudio periodismo tengo más capacidad para analizar las fuentes informativas", "nos lo ha enseñado en la universidad" o "estudiando periodismo aprendes a distinguir". En este sentido, podría considerarse cumplida la hipótesis de partida, según la cual, los estudiantes de periodismo están alerta y muestran preocupación por verificar la credibilidad de la información que consumen. Por otro lado, en opinión de los encuestados, los temas en lo que más abundan las fake news son la política y los temas sociales. 
Gráfico 2. Temática en la que se propagan más Fake News.

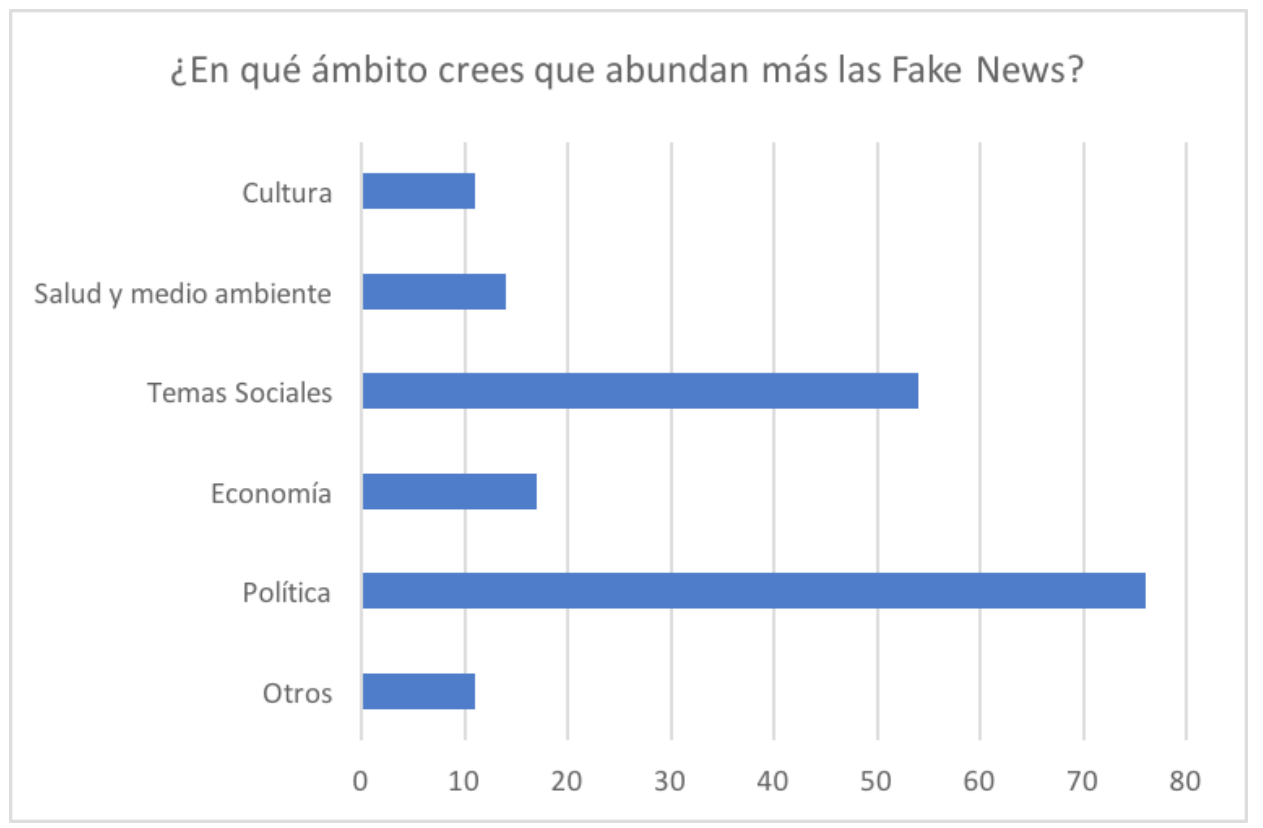

Fuente: elaboración propia

El 63\% se fía de las noticias que publican los medios de comunicación profesionales. Esa credibilidad no se la otorga a la prensa claramente el $4 \%$ que reconoce que no siempre se fía. El 33\% desconfía hoy en día de la información proveniente de los medios de comunicación. La mayoría de los encuestados considera, tal como se recoge en buena medida en la literatura existente en torno a la difusión de noticias falsas (Allcot y Gentzkow, 2017; Pariser, 2011), que son las redes sociales la vía que más propicia la propagación de las fake news, seguida del servicio de mensajería whatsapp.

Gráfico 3. Qué vía propaga más Fake News?

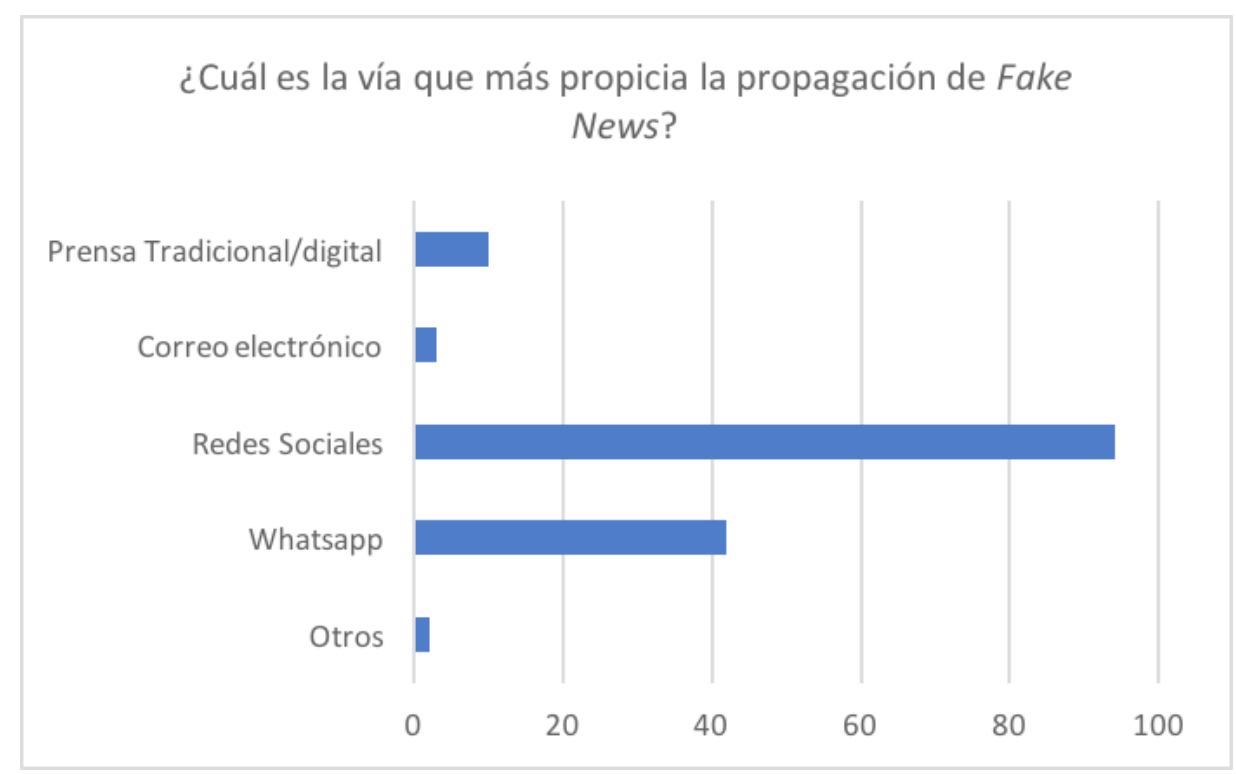

Fuente: elaboración propia ${ }^{6}$

\footnotetext{
${ }^{6}$ La opción prensa tradicional/digital del cuestionario se refiere a medios convencionales englobando a periódicos, radio y televisión.
} 
Respecto a la brecha generacional frente a las fake news, las opiniones están divididas casi al cincuenta por ciento. El 54\% de los encuestados opinan que tienen mayor criterio que sus padres o tutores para distinguir una información veraz de la que no lo es. Entre los que opinan esto, el motivo en algunos casos es que se consideran "más involucrados que sus padres en el mundo de las tecnologías y las redes sociales. Ellos (los padres) no usan redes sociales y tienden a pensar que "prácticamente todo lo que se comparte es verídico" o "se creen lo que les llega por Whatsapp". La condición de estudiantes de periodismo vuelve a surgir en las respuestas que se han registrado entre los que opinan que tienen mayor criterio que sus padres: "porque soy estudiante de periodismo, y ellos no", "porque hemos aprendido a contrastar las noticias en diferentes medios" o "lo veo desde un prisma más profesional".

Finalmente, un porcentaje amplio de los encuestados, el 91\%, cree que sería interesante que se implantaran cursos formativos en las escuelas para enseñar a distinguir la información fiable de la que no lo es.

\subsection{Resultados de la segunda fase}

Tal y como se recoge en el apartado de la metodología, una vez obtenidos los datos de la investigación principal que trataba de pulsar la opinión y la actitud de los estudiantes de periodismo, se procedió a ampliar el estudio a estudiantes de otras áreas de conocimiento relacionadas con los temas que son susceptibles de encajar en la temática de las fake news.

Los resultados de esta segunda fase arrojan, en cierto modo, resultados similares a las obtenidas en la primera fase. Es decir, las definiciones que los estudiantes de otras áreas hacen del concepto planteado, los medios a través de los que se informan, la percepción que tienen sobre su capacidad de detectar noticias falsas, etc. no distan de los obtenidos en la primera fase. De hecho, en el caso de los estudiantes de periodismo, un 82\% reconocía haberse creído alguna vez una información falsa, y este porcentaje es parecido entre los estudiantes de otras áreas, que ha registrado un 84,5\% (gráfico 4). Sin embargo, entre los estudiantes de periodismo, el $80 \%$ de los encuestados creían tener criterio para distinguir una información o noticia fiable de otra que no lo es. Esta misma pregunta entre los estudiantes de otras áreas ha arrojado un dato inferior, con un $67 \%$ por ciento de personas que afirman estar preparadas para detectar las noticias falsas.

Al ser este porcentaje menor que el obtenido en la primera fase, podemos decir que el alumnado de otras áreas de conocimiento no está tan preparado para hacer frente a la detección de Fake News, tal y como se afirmaba en la hipótesis de partida. Al menos se puede afirmar que es esa la percepción que tienen de su capacidad para discernir entre informaciones fiables y las que no lo son.

Gráfico 4. Comparativo entre estudiantes según especialidad (\%)

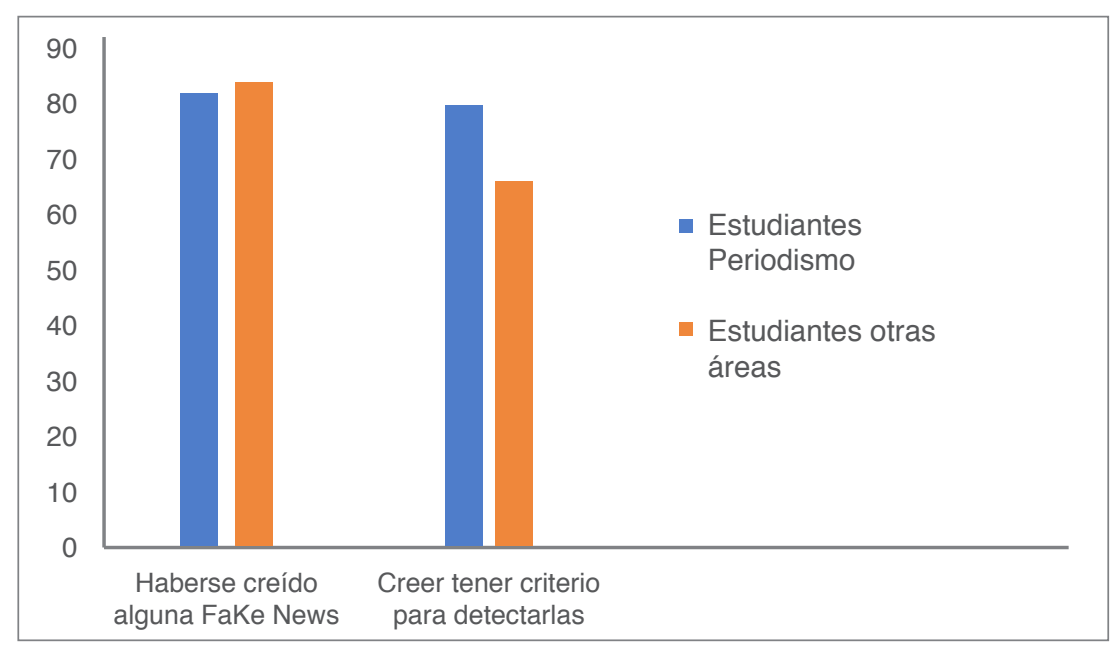

Fuente: elaboración propia 
Gráfica 5. Temática en la que se propagan más Fake News, según especialidad

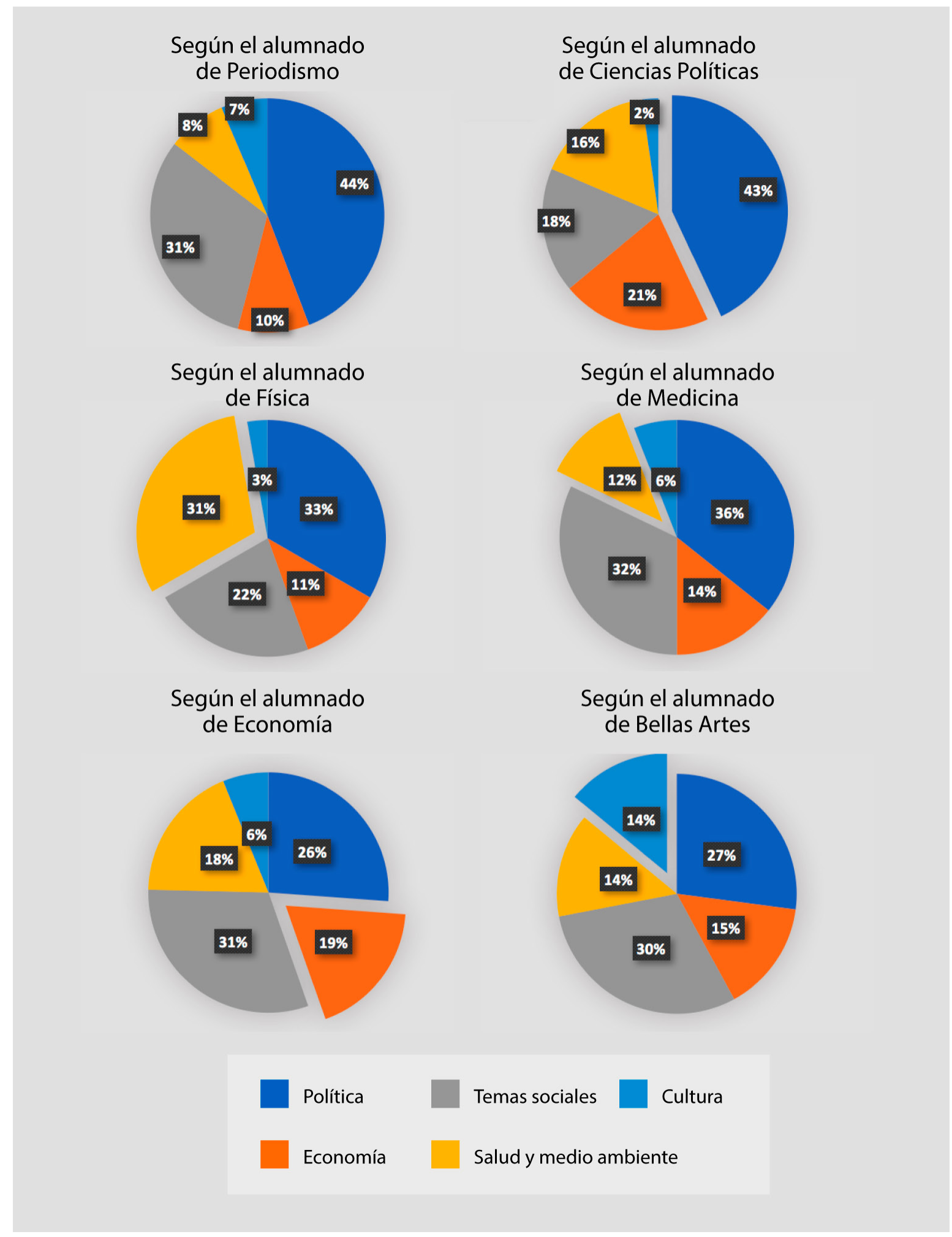

Fuente: Elaboración propia

Uno de los puntos donde se detecta mayor diferencia es en la pregunta que hace referencia a la temática principal de las Fake News. Ante la cuestión “¿Cuál es el ámbito donde más proliferan las Fake News?" todos coinciden en la política como el tema más afectado, pero las segundas, terceras, cuartas y quintas posiciones varían según el área de conocimiento. La hipótesis que planteaba la 
idea de que los estudiantes tendrían la percepción de que las Fake News son más recurrentes en los temas relacionados con su ámbito de estudio no se cumple del todo (aunque sí hay cierto grado de coincidencia).

Así, el alumnado del Grado de Física otorga una segunda posición a los temas relacionados con la salud y el medio ambiente, con un $31 \%$ por ciento de las respuestas, muy cerca del $33 \%$ que tiene el primer tema (política), cuando en la primera fase los estudiantes de periodismo relegaban la temática de salud y medio ambiente a una penúltima posición, con un $8 \%$.

Ocurre algo similar con los resultados obtenidos de los estudiantes de Economía y Bellas Artes. En el caso del alumnado de Economía, sin elevarlo hasta la primera posición, donde en todos los casos gana la política, han otorgado al tema económico un índice mayor que el resto, únicamente por detrás del que han registrado los estudiantes de Ciencias Políticas.

En el caso de los alumnos de Bellas Artes, la cultura ha registrado un 14\% de los votos, por encima del puntuado por los encuestados del resto de áreas de conocimiento. Sin embargo, el alumnado de Medicina ha dejado el tema de la salud y medio ambiente en el penúltimo lugar.

\section{Conclusiones}

Los resultados del presente estudio señalan un alto índice de alerta por parte de los estudiantes de periodismo, ante las denominadas Fake News. Gracias a su capacidad casi innata para manejar las nuevas tecnologías, no consideran que en el futuro vayan a encontrar muchas dificultades para gestionar grandes cantidades de información y para seleccionar aquellos datos que les resulten más verídicos. Esta capacidad de lidiar con bulos o noticias de dudosa credibilidad también la tienen asumida, aunque en menor medida (o así lo consideran) los estudiantes de otras áreas de conocimiento.

Tras examinar los resultados de las encuestas realizadas para este estudio, se detecta que, frente a los contenidos dudosos, buscan información "en diarios digitales que tienen más reputación y resultan más fiables". Aquí está el reto de los periodistas y diarios digitales en este mar de informaciones y datos que constituye internet: Resultar creíbles, transmitir credibilidad y construir reputación, frente a otros sitios web.

El alumnado apenas cita el correo electrónico como medio de propagación de fake news, al que gana el servicio de mensajería Whatsapp. Muchas de las cadenas falsas y bulos que actualmente se propagan por esta última vía difunden los mismos contenidos que las que se propagaban por correo electrónico. Parece que ha habido una continuidad en la propagación de ciertos bulos pero un traspaso de plataforma de difusión.

Dado que en la segunda fase de la investigación ha habido algunas diferencias en la respuesta sobre la temática donde más abundan las fake news, se ha detectado de forma sutil en estos resultados el fenómeno conocido popularmente como Baader-Meihof o la atención selectiva del entorno. Esto explicaría que los alumnos de Física tengan en consideración los temas de la salud o el medio ambiente como susceptibles de ser invadidos por Fake News, cuando es un tema que apenas contemplan los estudiantes de otras áreas de conocimiento; o que los alumnos de Economía eleven el porcentaje otorgado a la economía en comparación al resto; o que ocurra los mismo con los alumnos de Bellas Artes con el tema de la cultura. Sin embargo, no se ha registrado un patrón claro en este sentido, ya que los alumnos de medicina no coinciden con otorgar un alto índice al tema de la salud.

El hecho de alentar a los estudiantes, por parte del profesorado, a no compartir noticias falsas en las redes sociales puede ayudar a frenar la circulación de la desinformación. Estar bien informado sobre la actualidad, a través de los canales adecuados, también puede ayudar a los jóvenes a identificar noticias 
falsas. El primer paso para lograr estos objetivos es armarlos con habilidades de alfabetización mediática o habilidades que les permitan a los usuarios acceder, analizar y evaluar la información online. La necesidad de la formación en este sentido es uno de los puntos de la investigación donde mayor unanimidad se ha obtenido, en todas las áreas de conocimiento. Cabe apuntar que esta investigación ofrece fundamentalmente un análisis comparativo y descriptivo correspondiente a un contexto muy concreto, cuyas limitaciones nos invitan a seguir investigando en una línea metodológica más amplia y extrapolable, dado el interés que suscita el análisis de la percepción y la actitud de los jóvenes ante las Fake News.

\section{Bibliografía}

Alberto Notario, C. (2018). La generación del Milenio y su papel en el futuro de la inteligencia y la seguridad. En Nota de Futuro 1/2018. http://intranet.bibliotecasgc.bage.es/intranet-tmpl/prog/ local_repository/documents/documents/20576_21824.pdf

Allcott, H. y Gentzkow, M. (2017). Social media and fake news in the 2016 election. En The National bureau of economic research. https://pubs.aeaweb.org/doi/pdfplus/10.1257/ jep.31.2.211

Anderson, J., y Rainie, L. (2012). Millennials will benefit and suffer due to their hyperconnected lives. http://www.pewinternet.org/2012/02/29/millennials-will-benefit-and-suffer-due-totheirhyperconnected-lives/

Babbie, E. (1999). Técnicas de la investigación social. Thompson.

Banaji, Sh., \& Buckingham, D. (2013). The Civic Web. Young People, the Internet and Civic Participation. Cambridge, MA: The MIT Press.

Berinsky, A. (2017). Rumors and health care reform. Experiences in political misinformation. Bristish Journal of Political Science, 47 (2): 241-262.

Brandtzaeg, P., Folstad, A. \& Chaparro, M. A. (2017). How Journalists and Social Media Users Perceive Online Fact-Checking and Verification Services. Journalism Practice, 12(9), pp. 11091129. https://doi.org/10.1080/17512786.2017.1363657

Casero, A. (2009). Hacia la opinión pública 2.0. El Impacto del periodismo ciudadano sobre la esfera pública. En Medina, J., Rom, J.y Canosa, F. (eds). La Metamorfosis del espacio mediático. Trípodos.

Costera Meijer, I. (2007). The Paradox of Popularity; How Young People Experience the News. Journalism Studies, 8 (1), 96-116.

Del Barrio, A. y Ruiz, I. (2017). Hábitos de uso del whatsapp por parte de los adolescentes. INFAD Revista de Psicología, $\mathrm{N}^{\circ} 1$, Monográfico 1, 23-30.

Deuze, M. (2005). Mediawork. Cambridge, MA: Polity Press.

Drok, N. y Hermans, L. (2016). Is there a future for slow journalism? Journalism Practice, 10 (4), 539-554.

Fernández Cruz, F. J. y Fernández Díaz, M. J. (2016). Los docentes de la generación Z y sus competencias digitales. Comunicar, $\mathrm{N}^{\mathrm{o}}$ 46, 97-105.

Fisher, C. (2016). The trouble with 'trust' in news media. Communication Research and Practice, 2(4), 451-465. 
Foro Económico Mundial. (2017). Global Shapers Annual Survey 2017. Sharpers Survey 2017: http://www.shaperssurvey2017.org

Friggeri, A., Adamic, L.A. Eckles, D. and Cheng J. (2014). Rumor Cascades. Eighth International AAAI Conference on Weblogs and Social Media.

Gil, G. y Martínez, M. R (2001). Metodología de las Encuestas. En Navas, M.J. (Ed.). Métodos, Diseños y Técnicas de Investigación Psicológica. Madrid. UNED

Gottfried, J. \& Shearer, E. (2016). News Use Across Social Media Platforms 2016. Pew Research Center. Journalism \& Media. https://www.journalism.org/2016/05/26/news-use-across-socialmedia-platforms-2016/

Gottfried, J., \& Barthel, M. (2015). How Millennials' political news habits differ from those of Gen Xers and Baby Boomers, Pew Research Center. https://www.pewresearch.org/facttank/2015/06/01/political-news-habits-by-generation/

Guerrero Pérez, E. (2018). La fuga de los millennials de la televisión lineal. Revista Latina de Comunicación Social, 73, 1231-1246. http://www.revistalatinacs.org/073paper/1304/63es.html

Hidalgo Pérez, A. A. (2017). Nuevo escenario en la gestión del capital humano. Harvard Deusto Business Review, 268, 36-47.

Jones, A. (2008). Losing the news: The future of the news that feeds democracy. Oxford University Press.

Laufer, P. (2011). Slow News; A Manifesto for the Critical News Consumer. Oregon State University Press.

Leahy, K., McGinley, J., Thompson, J., \& Weese, T. (2011). Intelligence Community Assessment: Generational Difference in Workplace Motivation. Intelligence Reform and Transformation, 29(1), 1-16.

Lenhart, A., Purcel, K., Smith, A. \& Zickuhr, K. (2010). Social Media and movil Internet use among teens and young adults. Pew Internet and American life. https://files.eric.ed.gov/fulltext/ ED525056.pdf

López, A. (2011). Usos y actitudes de estudiantes universitarios futuros profesores sobre tecnologías de la información y la comunicación (TIC) y recursos sociales de internet. Questión, 31. http://sedici.unlp.edu.ar/handle/10915/34531

Magallón, R. (2016). El ADN de la Generación Z. Entre la economía colaborativa y la economía disruptiva. Revista de Estudios de Juventud, 114, 29-44.

Marchi, R. (2012). With Facebook, Blogs, and Fake News, Teens Reject Journalistic "Objectivity". Journal of Communication Inquiry, 36(3) 246-262.

Palomo, B. y Sedano, J. (2018). WhatsApp como herramienta de verificación de fake news. El caso de B de Bulo. Revista Latina de Comunicación Social, 73, 1384-1397. http://www. revistalatinacs.org/073paper/1312/71 es.html

Parra, P. \& Oliveira, L. (2018). Fake News: Una revisión sistemática de la literatura. Obserbatorio $(O B S), \mathrm{N}^{\mathrm{o}}$ extra $1,54-78$.

Parreira do Prado, M. (2019). La proliferación de las "fake news" y sus algoritmos daña la cultura democrática. Ámbitos. Revista Internacional de Comunicación, 45, 89-106. 
Pariser, E. (2011). The filter of bubble. Penguin Books.

Pew Research Center (2014). Millennials in Adulthood: Detached from Institutions, Networked with Friends. Pew Research Center. http://www.pewsocialtrends.org/2014/03/07/millennials-inadulthood/

Plazibat, I., Dadić, M., \& Petričević, D. (2017). Do the millennials make a difference in retail?. Theory and Applications in the Knowledge Economy, 186. https://www.researchgate. net/publication/322686268_Organizational_Culture_and_Leadership's_Impact_on_a_Safety_ Program_Change_Model

Spratt, H. E. \& Agosto, D. (2017). Fighting fake news because we all deserve the truth: Programming idea for teaching teens media literacy. Young Adult Library Services, 15 (4), 17-21.

Schroer, W. (2008). Defining, Managing and Marketing to Generation X, Y and Z. The Portal, 10, https://s3.amazonaws.com/rdcms-iam/files/production/public/newimages/portalpdfs/2008_03_04.pdf

Visauta, B. (1989). Técnicas de investigación Social. I: Recogida de datos. Promociones y Publicaciones Universitarias.

Wells, N. (2015). Who are the Millennials Anyway? CNBC. http://www.cnbc.com/2015/05/22/ who-are-the-millennials-anyway.html

Wineburg, S. \& McGrew, S. (2016). What Students Don't Know About Fact-Checking. Education Week Vol. 36, Issue 11, 22-28. http://odl.gmu.edu/wp-content/uploads/Why-Students-CantGoogle-Their-Way-to-the-Truth-Education-Week.pdf 